\title{
Plasmonic modulator based on gain-assisted metal-semiconductor-metal waveguide
}

\author{
Babicheva, Viktoriia E.; Kulkova, Irina V.; Malureanu, Radu; Yvind, Kresten; Lavrinenko, Andrei V.
}

Published in:

Photonics and Nanostructures - Fundamentals and Applications

Link to article, DOI:

10.1016/j.photonics.2012.05.008

Publication date:

2012

Link back to DTU Orbit

\section{Citation (APA):}

Babicheva, V. E., Kulkova, I. V., Malureanu, R., Yvind, K., \& Lavrinenko, A. V. (2012). Plasmonic modulator based on gain-assisted metal-semiconductor-metal waveguide. Photonics and Nanostructures - Fundamentals and Applications, 10(4), 389-399. https://doi.org/10.1016/j.photonics.2012.05.008

\section{General rights}

Copyright and moral rights for the publications made accessible in the public portal are retained by the authors and/or other copyright owners and it is a condition of accessing publications that users recognise and abide by the legal requirements associated with these rights.

- Users may download and print one copy of any publication from the public portal for the purpose of private study or research.

- You may not further distribute the material or use it for any profit-making activity or commercial gain

- You may freely distribute the URL identifying the publication in the public portal 


\title{
Plasmonic modulator based on gain-assisted metal-semiconductor-metal waveguide
}

\author{
Viktoriia E. Babicheva ${ }^{\text {a,b,*, Irina V. Kulkova }}{ }^{\text {a }}$, Radu Malureanu ${ }^{a}$, \\ Kresten Yvind $^{\text {a }}$, Andrei V. Lavrinenko ${ }^{\text {a }}$ \\ ${ }^{a}$ Department of Photonics Engineering, Technical University of Denmark, Ørsteds Plads, Bld. 343, DK-2800 Kongens Lyngby, Denmark \\ ${ }^{\mathrm{b}}$ Moscow Institute of Physics and Technology, Institutsky Pereulok 9, 141700 Dolgoprudny, Russia
}

Received 2 March 2012; received in revised form 4 May 2012; accepted 7 May 2012

\begin{abstract}
We investigate plasmonic modulators with gain material to be implemented as ultra-compact and ultra-fast active nanodevices in photonic integrated circuits. We analyze metal-semiconductor-metal (MSM) waveguides with InGaAsP-based active material layers as ultra-compact plasmonic modulators. The modulation is performed by changing the gain of the core, that results in different transmittance through the waveguides. A MSM waveguide enables high field localization and therefore high modulation speed. Bulk semiconductor, quantum wells and quantum dots, arranged in either horizontal or vertical layout, are considered as the core of the MSM waveguide. Dependences on the waveguide core size and gain values of various active materials are studied. The designs consider also practical aspects like $\mathrm{n}$ - and $\mathrm{p}$-doped layers and barriers in order to obtain close to reality results. The effective propagation constants in the MSM waveguides are calculated numerically. Their changes in the switching process are considered as a figure of merit. We show that a MSM waveguide with electrical current control of the gain incorporates compactness and deep modulation along with having a reasonable level of transmittance.
\end{abstract}

(C) 2012 Elsevier B.V. All rights reserved.

Keywords: Surface plasmons; Plasmonic waveguides; Metal-semiconductor-metal waveguides; Modulators; Semiconductor optical devices; Integrated circuits

\section{Introduction}

Nanoplasmonics is a booming focus area in nanophotonics with potential applications in sensing and integrated circuits $[1,2]$. However, until now the main obstacle in the implementation of plasmonic nanocomponents is the presence of high losses in connection with

\footnotetext{
* Corresponding author at: Department of Photonics Engineering, Technical University of Denmark, Ørsteds Plads, Bld. 343, DK-2800 Kongens Lyngby, Denmark. Tel.: +45 45256883; fax: +45 45936581.

E-mail addresses: vbab@fotonik.dtu.dk, baviev@gmail.com (V.E. Babicheva).
}

highly confined plasmonic waves propagation. Alternatives to the conventional noble metals, silver and gold, are desirable and have been proposed recently [3-6].

A way to compensate losses is the introduction of gain materials [7-9]. Recently, loss compensation by gain in hybrid dielectric loaded plasmonic waveguides has been studied [10,11]. Garcia-Blanco et al. [10] analyzed long-range dielectric-loaded surface plasmonpolariton waveguides and loss compensation by the rare-earth-doped double tungstate crystalline material as a gain medium. A hybrid plasmonic waveguide with a nano-slot was examined in [11], where also potential gain media were discussed. 
Nevertheless, hybrid dielectric loaded plasmonic waveguides suffer from optical confinement (device sizes are of the order of microns). The strongest mode confinement can be achieved in metal-insulator-metal (MIM) waveguides [12,13]. The MIM waveguide is a promising structure. It provides a base for extremely fast and efficient ultra-compact plasmonic devices, including modulators [14,15], photodetectors [16] and lasers [17]. However, there is a trade-off on applications: the MIM waveguide allows high fields localization, but by contrast, propagation losses in the waveguide are also high.

Loss compensation using a gain semiconductor core in MIM waveguides led to the concept of metalsemiconductor-metal (MSM) waveguide. Gain-assisted propagation in a subwavelength MSM waveguide was theoretically analyzed [9]. The thickness of the active core of high-index semiconductor was varied from $50 \mathrm{~nm}$ to $500 \mathrm{~nm}$. For a gold MSM waveguide the required critical gain coefficient for a lossless propagation is achievable at telecommunication wavelengths. More recent systematic theoretical investigation of giant modal gain and amplified propagation in MSM structures with silver plates and the 100-200 nm semiconductor cores is presented in [18]. The existence of an optimum gain level which gives the strongest plasmonic resonance and slowing down of the group velocity is demonstrated.

Encapsulation of InP-based semiconductors in metal and configuring compact MSM waveguides was discussed regarding lasing application. Lasing in metalliccoated nanocavities, namely in the MSM waveguide with rectangular cross-section $\mathrm{InP} / \mathrm{InGaAs} / \mathrm{InP}$ pillars, is realized [19-21]. Theoretical analysis of a MSM waveguide with the InGaAs core [22] shows the possibility of realization of a plasmonic semiconductor nano-laser. Semiconductor electrons-holes dynamics in conduction and valence bands is described numerically. The influence of intermediate low index buffer layers in a MSM sandwich is studied as well.

One of the hottest topics is active plasmonics, which combines semiconductor electronics and nonlinear optics to control the optical properties of different nanodevices [23,24]. Recently an all-semiconductor active plasmonic system based on InAs heterostructures has been proposed for mid-infrared operation wavelengths [25]. An InGaAsP quantum wells stack was used to improve properties of negative index materials $[26,27]$. Whereas the goal of the work was to design the fast optical modulation (tens of picoseconds time scale) in the $\mathrm{Si}$ spacer layer of such metamaterials, gain produced rather low changes in transmission because of metal screening.
From another point of view, changing the permittivity and thus controlling the propagation in a MIM waveguide is an attractive subject of active plasmonics. A straightforward way is to vary the transmission through the waveguide by changing the permittivity of a sandwiched medium. This idea led to theoretical description of switches using gain-assisted MIM structures $[28,29]$, where the proposed solutions utilize a quantum-dots-doped semiconductor. It is also shown that Fabry-Perot resonances in the active core layer enhance the switching effect.

Another possibility to control propagation of light in a MIM waveguide by changing permittivity of the medium in side-coupled cavities was theoretically analyzed in [28,30-32]. In particular, it was shown that implementation of gain media in parts of the waveguide can compensate losses along the device [30].

Despite the fact that photonic switches based on either bulk semiconductors or quantum dots have been studied, there is a lack of comprehensive research on MSM compact devices with the active core from welldefined materials. All proposed MSM structures with loss compensation and switching are analyzed in the assumption of some model gain material uniformly distributed along the dielectric core. Here we bring the subject closer to reality considering InGaAsP-based semiconductors in the MSM core.

We study the performance of a MSM device for plasmonic switching applications. In particular, ultracompact modulator can be designed. The semiconductor core is considered consisting of a bulk gain medium, quantum wells or layers with quantum dots. We examine several potentially realizable designs of structures and analyze modulator's performance. In Section 2 we describe our theoretical model and numerical approach to MSM waveguide simulations. In Section 3 we analyze possible MSM waveguide arrangement. Performance of device with the bulk semiconductor core is studied in Section 4, with quantum-dots-based core in Section 5 and with quantum wells layers in Section 6. Discussion and final sum up of the results are presented in Section 7.

\section{Simulation model}

We performed frequency domain simulations using the commercial software package CST Microwave Studio [33]. The material gain $g$ is connected with the imaginary part of the semiconductor permittivity: $\varepsilon^{\prime \prime}=-g n^{\prime} / k_{0}$, where $k_{0}$ is the free-space wave-number and $n^{\prime}$ is the real part of the refractive index. So, for positive gain values the imaginary part of permittivity is 
negative. Due to the CST constrains, materials with the negative imaginary part of permittivity cannot be implemented directly. To circumvent this constrain and perform CST numerical simulations with gain materials we follow the suggestion from [34]. It consists in editing the permittivity values, in particular changing the sign of $\varepsilon^{\prime \prime}$, in the Visual Basic script of the history list file. CST simulations were compared with analytical calculations to validate this approach.

Fabry-Perot resonances on a finite-length semiconductor core can significantly increase the effect of the total transmission change in a MSM waveguide (see e.g. [28,29]). Partial removing of lossy material from waveguide's core can increase transmittances as well $[35,36]$. However, fabrication of a patterned core inside MSM is challenging and we do not consider it here. So we imply that plasmonic waveguides are uniform in the propagation direction and study device characteristics independently from its length.

We are interested in transmission properties of MSM structures at the telecom wavelength $1.55 \mu \mathrm{m}$. A MSM waveguide supports a surface plasmon polariton (SPP) wave in the transverse magnetic (TM) polarization. The silver plates (Fig. 1) have the thickness of $120 \mathrm{~nm}$, which is enough to keep the domain-termination error at negligible level. The silver permittivity is approximated by the Drude formula:

$\varepsilon_{\mathrm{Ag}}=\varepsilon_{\infty}-\frac{\omega_{\mathrm{pl}}^{2}}{\omega^{2}+i \gamma \omega}$,

where $\varepsilon_{\infty}=1$, plasma frequency $\omega_{\mathrm{pl}}=1.38 \times 10^{16} \mathrm{~s}^{-1}$ and collision frequency $\gamma=3.22 \times 10^{13} \mathrm{~s}^{-1}$. It gives $\varepsilon_{\mathrm{Ag}}=-128.7+3.44 i$ at $\lambda=1.55 \mu \mathrm{m}$.

Signal attenuation (extinction) per device's unit length can be defined as

$A_{/ L}=\frac{10 \lg \left(P_{0} / P\right)}{L}=8.68 \cdot \operatorname{Im}\left(k_{\mathrm{eff}}\right)$,

while logarithmic extinction ratio (ER) per unit length is

$$
\begin{aligned}
\mathrm{ER}_{/ L} & =\frac{10 \lg \left(P_{\text {on }} / P_{\text {off }}\right)}{L} \\
& =8.68 \cdot\left(\operatorname{Im}\left(k_{\text {eff }}\right)_{\text {off }}-\operatorname{Im}\left(k_{\text {eff }}\right)_{\text {on }}\right),
\end{aligned}
$$

where $\operatorname{Im}\left(k_{\text {eff }}\right)_{\text {on }}$ and $\operatorname{Im}\left(k_{\text {eff }}\right)_{\text {off }}$ are the imaginary parts of the effective propagation constant of a waveguide mode in the on-state (voltage or electrical current is switched on) and off-state (voltage or current is switched off). ER shows how strong we can vary the mode propagation through the waveguide.

However, for plasmonic switching devices, that possess significant propagation losses, another length
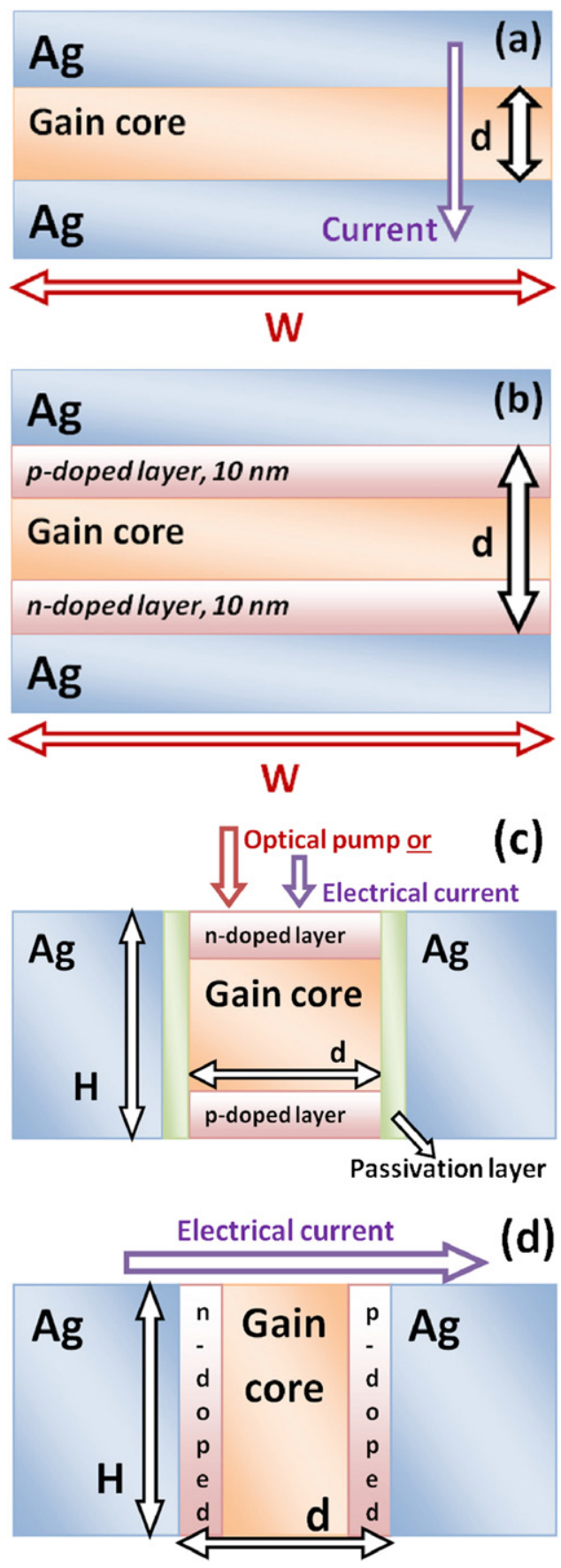

Fig. 1. Cross sections of MSM waveguides with the gain core. (a) Basic MSM waveguide. Horizontal arrangement. (b) Refined structure of the MSM waveguide, which includes the gain core and n- and p-doped layers. Horizontal arrangement. (c) Vertical arrangement with top pumping. (d) Vertical arrangement with metal plates as electrodes. 
independent figure of merit (FoM) can be defined (similar to $[15,35]$ ):

$\mathrm{FoM}=\frac{\mathrm{ER}_{/ L}}{A_{/ L}}=\frac{\left|\operatorname{Im}\left(k_{\mathrm{eff}}\right)_{\mathrm{on}}-\operatorname{Im}\left(k_{\mathrm{eff}}\right)_{\mathrm{off}}\right|}{\operatorname{Im}\left(k_{\mathrm{eff}}\right)_{\text {state }}}$,

where denominator is either $\operatorname{Im}\left(k_{\text {eff }}\right)_{\text {on }}$ or $\operatorname{Im}\left(k_{\text {eff }}\right)_{\text {off }}$ depending on which state is transmitting. FoM (4) describes how strong we can vary the mode propagation through the waveguide in comparison with the attenuation in transmitting state. ER or FoM should be chosen depending on particular waveguide properties. The high gain core in a MSM device can completely compensate plasmonic losses and cause wave amplification. In case of $\operatorname{Im}\left(k_{\text {eff }}\right)_{\text {on }} \cong 0$ we can receive infinitely high FoM (4), that does not have a physical meaning. Most of structures studied in the paper allow complete loss compensation. Therefore, characterizing MSM devices we present results for $\mathrm{ER}_{/ L}(3)$. Spontaneous emission noise is not included in our model.

The on-state refers to a MSM waveguide whose core (an InGaAsP-based semiconductor layer) is active and exhibits gain. Such active material can be designed to have a band gap at the wavelength of interest $1.55 \mu \mathrm{m}$. In the off-state the core material exhibits high losses interpreted as negative material gain. Zero-current negative gain is equal to the maximum material gain that can be theoretically achieved in the semiconductor material [37].

We also compared switching to an "insulator-state" of a MSM waveguide, which refers to the waveguide with a passive sandwiched medium. The passive medium means that the medium has a band gap at wavelengths shorter than the wavelength of interest $(1.55 \mu \mathrm{m})$. So precisely at $1.55 \mu \mathrm{m}$ it is transparent, i.e. it has neither gain nor loss caused by electron-hole recombination. Plasmonic related losses exceed those of the semiconductor core by far. So, in the "insulatorstate", the MSM device has losses caused by the silver electrodes only.

Depending on the active region structure, i.e. bulk semiconductor, quantum wells (QWs) or quantum dots (QDs), and carrier density the spectral range of gain of the InGaAsP-based material can vary from tens to hundreds of nanometers around the telecom wavelengths. This characteristic influences the device operation bandwidth. The energy band gap of the gain medium is controlled by the fraction of each component in the $\operatorname{In}_{x} \mathrm{Ga}_{1-x} \mathrm{As}_{y} \mathrm{P}_{1-y}$ material, and therefore defines the wavelength of the maximum gain. Material should also be engineered based on operating carrier density. Components with higher bandgap, which is closer to wavelength of interest $1.55 \mu \mathrm{m}$, should be used for lower carrier density and consequently achievable gain (e.g. $\mathrm{In}_{0.558} \mathrm{Ga}_{0.442} \mathrm{As}_{0.95} \mathrm{P}_{0.05}$ has bandgap $\sim 1.58 \mu \mathrm{m}$ ). However, we took data from [37] and studied bulk gain material $\operatorname{In}_{0.53} \mathrm{Ga}_{0.47} \mathrm{As}$ that has bandgap $\sim 1.69 \mu \mathrm{m}$ and requires a high carrier density to achieve high gain at $1.55 \mu \mathrm{m}$.

The infrared refractive index for different $\operatorname{In}_{x} \mathrm{Ga}_{1}$ ${ }_{-x} \mathrm{As}_{y} \mathrm{P}_{1-y}$ structures is approximately $n^{\prime}=3.1+0.46 y$ [38] and varies only slightly with respect to the wavelength. Therefore, we neglected the material dispersion.

In order to simulate an as-realistic-as-possible system we included the n- and p-doped layers at the top and bottom of the active layer (see Fig. 1b) to prevent the non-equilibrium distributions and recombination of carriers at the contacts. The $\mathrm{n}$ - and p-doped layers should have somewhat different composition to obtain the band gap at higher frequency. However, in calculations we fix the real part of permittivity $\varepsilon^{\prime}=12.46$ (which corresponds to the real part of refractive index $n^{\prime}=3.4-3.5$ for a small gain) for all studied semiconductors apart from permittivity of the InP layer fixed as $\varepsilon_{\text {InP }}=10$, which corresponds to $n_{\text {InP }}=3.16$. The imaginary part of effective propagation constant $\operatorname{Im}\left[k_{\text {eff }}\right]$ and modal gain $g_{\mathrm{m}}$ in the waveguide are proportional to each other: $g_{\mathrm{m}}=-2 \operatorname{Im}\left[k_{\mathrm{eff}}\right]$.

\section{MSM waveguide arrangements}

Realization of MSM waveguides based devices encounters several problems and can be accomplished in different ways. One way is to deposit metal plates as horizontal layers (Fig. 1a) and most of the fabricated devices are based on this procedure (e.g. $[13,14,16]$ ). However, InGaAsP-based heterostructures can be deposited only on a semiconductor native substrate, what means that some additional techniques, e.g. bonding or membrane etching and coating, should be applied to encapsulate semiconductor layers in metal. In such horizontal design n- and p-doped layers should be deposited between metal plates and gain medium while metal plates serve as electrodes. The horizontal layout is preferable for systems when a very narrow gap between metal plates is required. MSM waveguide can have the width of about $w \sim 1 \mu \mathrm{m}$ (see Fig. 1a). Nevertheless we assumed that the border influence is negligible thus simulated the system as a twodimensional one (i.e. $w$ is taken as infinitely long). However, as the noble metals exhibit poor adhesion to III-V semiconductor materials this possibility may prove to be a challenge to fabricate without using e.g. Ti 
or $\mathrm{Cr}$ adhesion layers. Such lossy layers can significantly affect device operation.

The other option is to etch narrow ridges and to coat them with metal. This technique is applied in the case of fabrication of nanolaser structures [20,22]. In such vertical design, the high aspect ratio (that is $H / d$, see Fig. 1c) is challenging to achieve in practice, despite the InP-based materials are relatively strong because of their crystalline structure. The high aspect ratio is required due to the mode confinement necessities. Because of finite aspect ratio $H / d$ the mode profile in such waveguide deviates from the two-dimensional case. Two cases can be considered here (Fig. 1c and d). The structure shown in Fig. 1c with very high aspect ratio was realized for lasing by Hill et al. [20]: the core thickness was $d=100-350 \mathrm{~nm}$, while the whole ridge height $H>1 \mu \mathrm{m}$. In Fig. 1c n- and p-doped layers are horizontal and can be deposited during the growth of the gain medium. In this case, metal plates do not serve as electrodes anymore and passivation layers (e.g. $\mathrm{Si}_{3} \mathrm{~N}_{4}$ or InP) on both sides of the ridge are required. In the vertical arrangement one can use either optical or electrical pumping. That assumes large distance of current propagation and can slow down the modulation. Electrically driven fast modulation can be realized in the design of Fig. 1d, which requires n- and p-doped layers depositing after ridge etching. However, it is very challenging for practical realization.

We performed numerical simulations of a MSM waveguide of finite height $H$ and various core thicknesses $d$ (corresponds to Fig. 1c in the passive state). Fig. 2 shows propagation length $L(L=1 /$ $\left.\operatorname{Im}\left[k_{\text {eff }}\right]\right)$ for different waveguides in comparison with

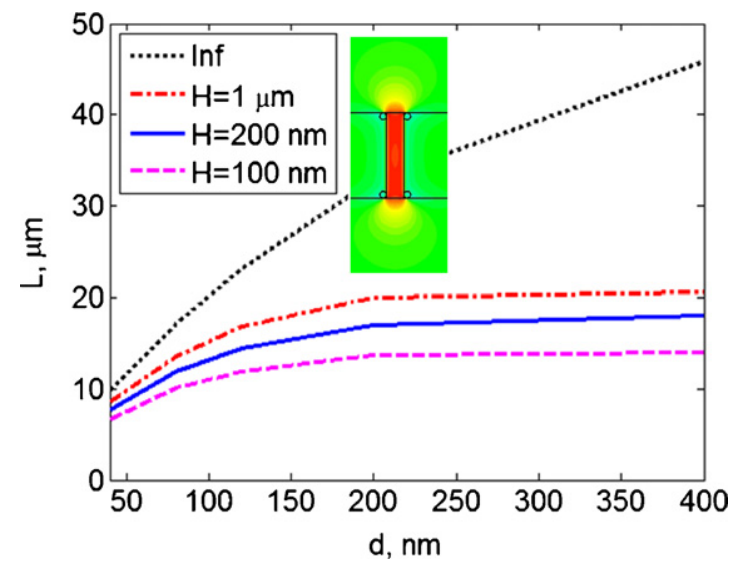

Fig. 2. Propagation length for MSM waveguide of finite height $H$ in passive state. Inset: electric field distribution for waveguide's cross section with $H=200 \mathrm{~nm}$ and $d=40 \mathrm{~nm}$. the ideal case (infinitely high waveguide). The waveguide is sandwiched between high-index material, the same as the core $(\varepsilon=12.46)$; metal corners are rounded for simulation reason with radius of curvature $4 \mathrm{~nm}$. Even for $H=1 \mu \mathrm{m}$ the propagation length halves because of mode spreading outside waveguide (see inset of Fig. 2).

\section{MSM waveguide with bulk gain medium}

In the first approximation the semiconductor core can be considered as a layer of uniformly distributed gain material (Fig. 1a). For the bulk gain core we set material gain $g_{\mathrm{b}}$ in the range from $-0.6 \times 10^{4} \mathrm{~cm}^{-1}$ to $0.6 \times 10^{4} \mathrm{~cm}^{-1}$ for $\operatorname{In}_{0.53} \mathrm{Ga}_{0.47}$ As composition at the telecom wavelength (equivalent to $\varepsilon^{\prime \prime}=0.5225$ to -0.5225 ) with carrier density up to $6.7 \times 10^{19} \mathrm{~cm}^{-3}$ [37]. The core thickness $d$ is varied from $20 \mathrm{~nm}$ to $400 \mathrm{~nm}$. The main advantage of the double-sided metalclad waveguide is the strong field localization between the plates. The inset in Fig. 3 shows the effective refractive index of the waveguide, growing from the refractive index of sandwiched material $n^{\prime}=3.53$ for a wide core up to 6.7 for a very thin core.

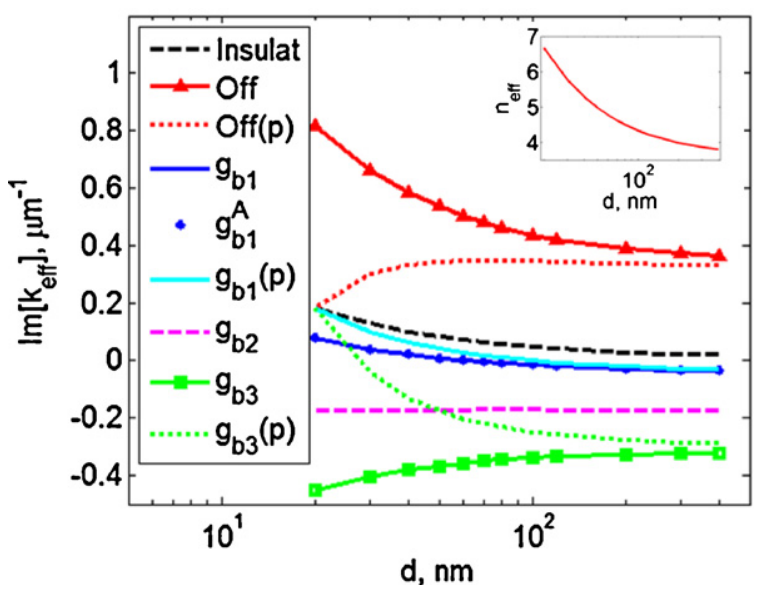

Fig. 3. Imaginary part of the effective propagation constant for various thicknesses of the bulk gain semiconductor core. All results are from CST numerical simulations apart from " $g_{\mathrm{b} 1} \mathrm{~A}$,", which is the analytical solution of the SPP dispersion relation for a three-layer system. Notation: "Insulat" for passive waveguide (insulator-state); "Off" for switched off current $g_{\mathrm{b}}=-0.6 \times 10^{4} \mathrm{~cm}^{-1}$; "Off(p)" the same for refined structure with n- and p- doped layers; " $g_{\mathrm{b} 1}$ " on-state with $g_{\mathrm{b} 1}=0.1 \times 10^{4} \mathrm{~cm}^{-1}$; " $g_{\mathrm{b} 1} \mathrm{~A}$," for analytical calculation same as " $g_{\mathrm{b} 1}$ "; " $\mathrm{g}_{\mathrm{b} 1}(\mathrm{p})$ " on-state with $g_{\mathrm{b} 1}=0.1 \times 10^{4} \mathrm{~cm}^{-1}$ in refined structure with n- and p- doped layers; " $g_{\mathrm{b} 2}$ " on-state with $g_{\mathrm{b} 2}=0.34 \times 10^{4} \mathrm{~cm}^{-1} ;$ " $g_{\mathrm{b} 3}$ " on-state with $g_{\mathrm{b} 3}=0.6 \times 10^{4} \mathrm{~cm}^{-1}$; " $g_{\mathrm{b} 3}(\mathrm{p})$ " on-state with $g_{\mathrm{b} 3}=0.6 \times 10^{4} \mathrm{~cm}^{-1}$ and refined structure with $n$ - and p-doped layers. Inset: effective refractive index in the MSM waveguide filled with $\operatorname{In}_{0.53} \mathrm{Ga}_{0.47} \mathrm{As}$ bulk gain medium. 
Fig. 3 shows $\operatorname{Im}\left[k_{\text {eff }}\right]$ versus the MSM core thickness for various gain values. We consider: the "insulatorstate" for the passive semiconductor core, "off-state" for the maximum loss in semiconductor as well as several gain values. To validate the CST simulations with the negative imaginary part of permittivity we compare results with the ones obtained by analytical solution of the SPP dispersion equation for a three-layer system (Fig. 3). The results match perfectly.

As was discussed above the system has very high losses in the off-state. With the increase in current, the material gain becomes higher and, at some point, compensates losses. For example, for the gain value $g_{\mathrm{b} 1}=0.1 \times 10^{4} \mathrm{~cm}^{-1}\left(\varepsilon^{\prime \prime}=-0.0871\right)$ complete loss compensation in the MSM waveguide $\left(\operatorname{Im}\left[k_{\text {eff }}\right]=0\right)$ can be achieved in the case of a core thickness $d \approx 60 \mathrm{~nm}$. Further increase of gain gives light amplification in such system. For gain values more than $g_{\mathrm{b} 2}=0.34 \times 10^{4} \mathrm{~cm}^{-1}\left(\varepsilon^{\prime \prime}=-0.2961\right)$ the strong field localization within the gap between metal plates starts to play a crucial role and the MSM design gives an essential benefit. In the case of high gain the $\operatorname{Im}\left[k_{\text {eff }}\right]$ dependency on the core thickness is monotonically increasing, opposite to the case of lower gain (see Fig. 3). In other words, for high gain values the thinner the MSM core the longer propagation can be achieved.

In Fig. 4 we show $\operatorname{Im}\left[k_{\text {eff }}\right]$ in the MSM waveguide with a bulk gain medium for various carrier densities. For better comparison, we converted the material gain data [37] into the semiconductor permittivity and added in Fig. 4 (dotted line). The imaginary part of semiconductor permittivity has nearly linear behavior on the logarithmic scale as follows from carriers and photons

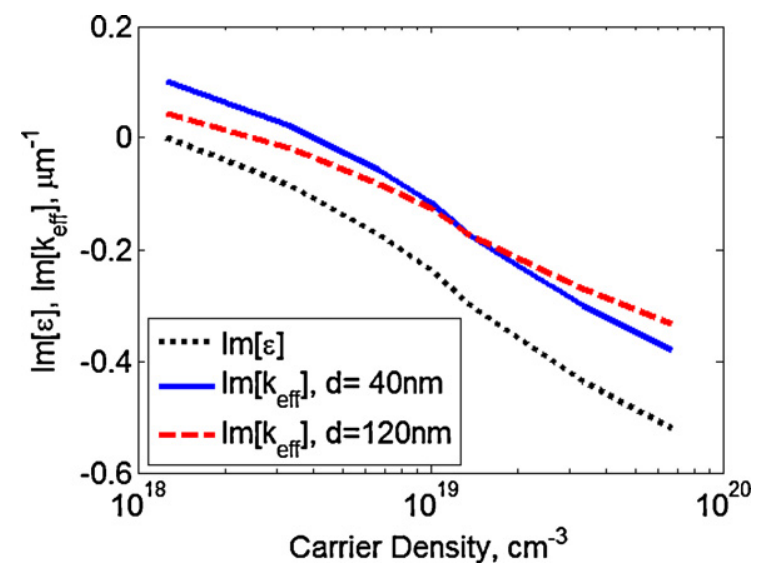

Fig. 4. Imaginary part of the effective propagation constant in the MSM waveguide with the bulk gain medium for various carrier densities. Imaginary part of semiconductor permittivity is shown for comparison. rate equations, thus the imaginary part of the effective propagation constant exhibits the same tendency.

Further we consider an advanced structure, which includes $n$ - and $\mathrm{p}$-doped layers on both sides of the active layer (see Fig. 1b). We assume the n- and p-doped layers are being $10 \mathrm{~nm}$ thick. These intermediate layers are approximated as insulator layers $\left(\varepsilon^{\prime \prime}=0\right)$ having the same permittivity as the semiconductor core, $\varepsilon^{\prime}=12.46$. The partial removal of the gain material causes an increase in $\operatorname{Im}\left[k_{\text {eff }}\right]$. In such MSM waveguides complete loss compensation with gain value $g_{\mathrm{b} 1}=0.1 \times$ $10^{4} \mathrm{~cm}^{-1}$ can be achieved for core thicknesses more than $100 \mathrm{~nm}$ (see Fig. 3).

Calculated ER of both systems versus the core thickness is shown in Fig. 5 for various transition states. Introduction of $\mathrm{n}$ - and $\mathrm{p}$-doped layers for thin core waveguides decreases ER, as expected.

As an additional option we also consider the difference between the on-state and passive state ("insulator-state") of the MSM waveguide. The "insulator-state", that is $g=0$, can be achieved in the studied material for relatively low carrier density, namely $1.25 \times 10^{18} \mathrm{~cm}^{-3}$ [37].

\section{MSM waveguide with quantum dots}

The second realistic option for the core design in MSM structures can be an InGaAsP layer with InAs QDs. Significant progress in QDs devices has been done at $1.3 \mu \mathrm{m}$ wavelength $[39,40]$ while efficient emission

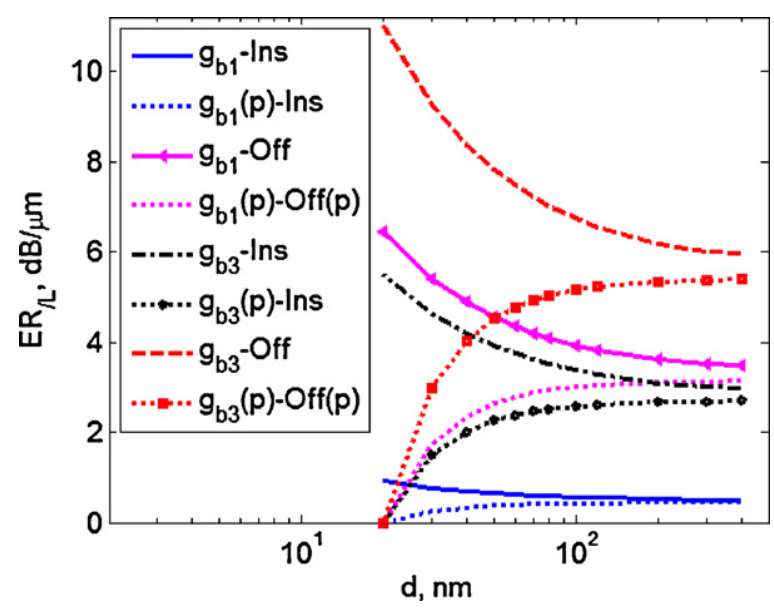

Fig. 5. ER of MSM device with the bulk gain core. Notation: " $g_{\mathrm{b} i}$ " Ins" shows switch between insulator-state with $g=0$ and on-state with $g_{\mathrm{b} i}\left(g_{\mathrm{b} 1}=0.1 \times 10^{4} \mathrm{~cm}^{-1}\right.$ and $\left.g_{\mathrm{b} 3}=0.6 \times 10^{4} \mathrm{~cm}^{-1}\right)$; “ $g_{\mathrm{b} i}(\mathrm{p})-\mathrm{Ins} "$ the same, but for the refined structure; " $g_{\mathrm{b} i}$-Off" shows switch between off-state with $g_{\mathrm{b}}=-0.6 \times 10^{4} \mathrm{~cm}^{-1}$ and on-state with $g_{\mathrm{b} i}$ $\left(g_{\mathrm{b} 1}=0.1 \times 10^{4} \mathrm{~cm}^{-1}\right.$ and $\left.g_{\mathrm{b} 3}=0.6 \times 10^{4} \mathrm{~cm}^{-1}\right) ; " g_{\mathrm{b} i}(\mathrm{p})-\mathrm{Off}(\mathrm{p}) "$ the same, but for the refined structure. 
on $1.55 \mu \mathrm{m}$ wavelength is still under investigation [41,42].

Despite on the small volume, QDs exhibit material gain more than one order of magnitude larger than QWs under the same injection current density because of the strong confinement and quantization of energy levels $[43,44]$. However, such gain can be obtained only when the electric field is in the QDs plane. Thus, in the horizontal design (Fig. 6a) QDs layer gives quite low gain, e.g. material gain $g_{\mathrm{TM}}=0.4 \times 10^{3}-1.2 \times$ $10^{3} \mathrm{~cm}^{-1}$ in conversion of the QDs to an equivalent bulk layer [45]. Such low bulk gain we discussed in the previous section. Therefore depositing of QDs in horizontal design does not provide any additional benefits.

Further we consider the system in Fig. 6b. The regular vertical arrangement of QDs grown by metalorganic vapor-phase epitaxy of columnar QDs (vertically aligned, closely stacked QDs) [45] by Stranski-Krastanov method [46].

Parameters for numerical simulations of QDs are the following. A single QD has an ellipsoid shape; the inplane cross-section is circular with $30 \mathrm{~nm}$ diameter, while the shortest ellipsoid axis is $5 \mathrm{~nm}$. QDs are arranged in a regular array with period $50 \mathrm{~nm}$ in both inplane directions. The described geometrical arrangement corresponds to $4 \times 10^{10} \mathrm{~cm}^{-2}$ QDs density [41]. An InGaAsP matrix containing one QDs layer has thickness $s=10 \mathrm{~nm}$ and $\varepsilon^{\prime}=12.46$, the same as in the

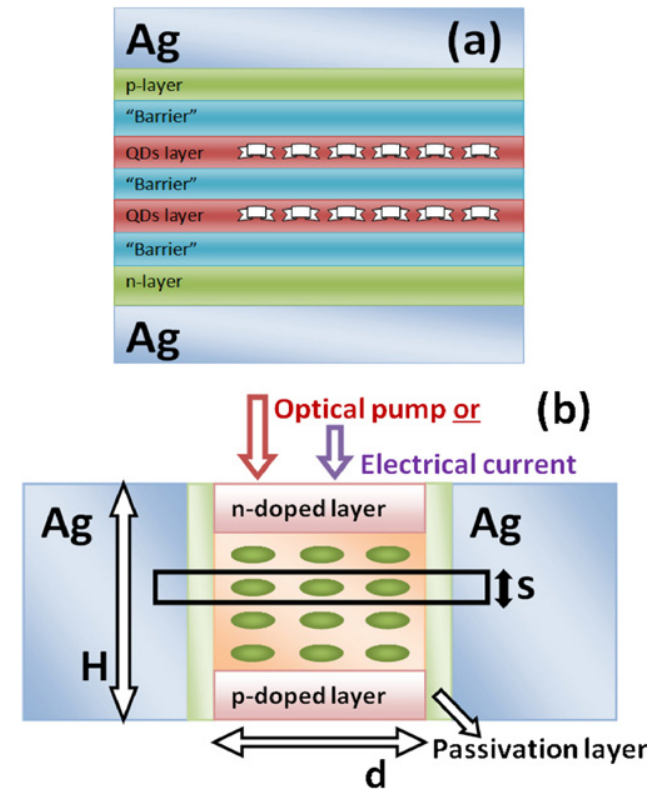

Fig. 6. Cross sections of the MSM waveguide with QDs layers. (a) Horizontal design. (b) Vertical design. previous analysis. We performed simulations for a single QDs layer with periodic boundary conditions in vertical directions, that is equivalent to the twodimensional waveguide with an infinite number of QDs layers. We used $g_{\mathrm{d} 1}=1 \times 10^{4} \mathrm{~cm}^{-1}$ and $g_{\mathrm{d} 2}=5 \times 10^{4} \mathrm{~cm}^{-1}$.

The QDs volume ratio in the 10-nm thick stack layer is approximately $9 \%$. For simulations we consider the equivalent system with bulk semiconductor (see Fig. 1a) having the gain value defined by the volumetric ratio and gain of the QDs. Thus, the average gain is $b_{\mathrm{d} 1}=0.9 \times 10^{3} \mathrm{~cm}^{-1}$ and $b_{\mathrm{d} 2}=4.5 \times 10^{3} \mathrm{~cm}^{-1}$.

The total thickness of semiconductor between the metal plates $d=50 \mathrm{Nm}$, where $N=1-5$ is a number of columns. Up to 5 columns containing QDs were considered for simulations. The imaginary part of the effective propagation constant and ER of MSM devices with QDs are shown in Figs. 7 and 8, respectively. Results for QDs and an effective bulk medium are perfectly matched, so the averaging procedure is justified. We assume that the perfect periodic arrangement of QDs in simulations does not influence system propagation properties, which are the same as for a core with randomly distributed QDs. While to achieve $b_{\mathrm{d} 2}=4.5 \times 10^{3} \mathrm{~cm}^{-1}$ for bulk material requires a very high current, it is feasible for the QDs effective medium.

For the highest gain $g_{\mathrm{d} 2}=5 \times 10^{4} \mathrm{~cm}^{-1}$ in the onstate, the value of $\operatorname{Im}\left[k_{\text {eff }}\right]$ is monotonically increasing with the number of columns, which causes decreasing of ER. Average gain $b_{\mathrm{d} 2}=4.5 \times 10^{3} \mathrm{~cm}^{-1}>g_{\mathrm{b} 2}$, so we observe the same trend in $\operatorname{Im}\left[k_{\text {eff }}\right]$ versus size dependence as for the bulk material (see Fig. 3).

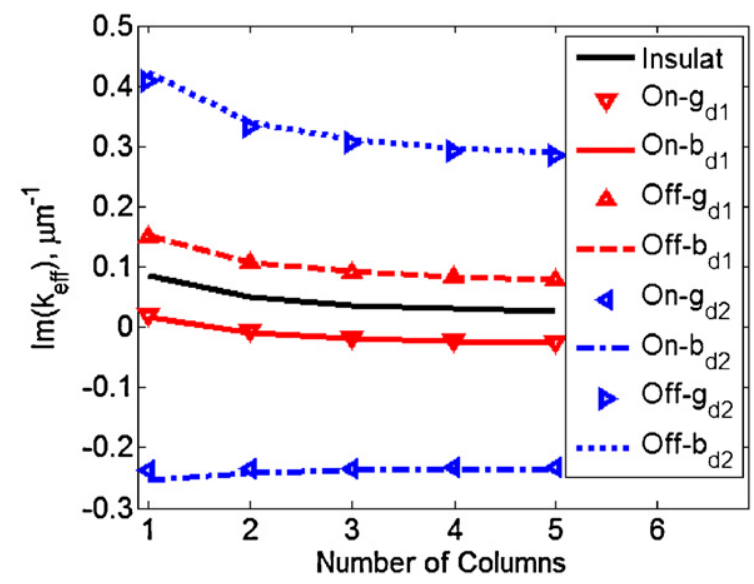

Fig. 7. Imaginary part of the effective propagation constant in the MSM waveguide with QDs. Notation: "Insulat" is passive waveguide, $g_{\mathrm{d} 1}=1 \times 10^{4} \mathrm{~cm}^{-1}, g_{\mathrm{d} 2}=5 \times 10^{4} \mathrm{~cm}^{-1}, " b_{\mathrm{d} 1}$ " and " $b_{\mathrm{d} 2}$ " corresponds to the MSM waveguide with uniform distribution of bulk gain material with $b_{\mathrm{d} 1}=0.9 \times 10^{3} \mathrm{~cm}^{-1}$ and $b_{\mathrm{d} 2}=4.5 \times 10^{3} \mathrm{~cm}^{-1}$. 


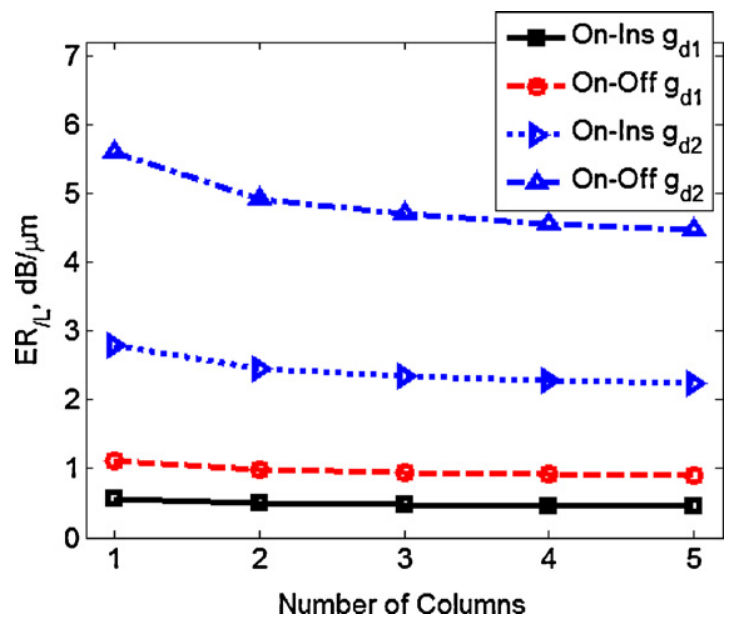

Fig. 8. ER of the MSM device with QDs. "On-Ins $g_{\mathrm{d} i}$ " shows transition between insulator-state $(g=0)$ and on-state with $g_{\mathrm{d} i}$. "On-Off $g_{\mathrm{d} i}$ " shows transition between off-state with $-g_{\mathrm{d} i}$ and onstate with $g_{\mathrm{d} i}\left(g_{\mathrm{d} 1}=1 \times 10^{4} \mathrm{~cm}^{-1}\right.$ and $\left.g_{\mathrm{d} 2}=5 \times 10^{4} \mathrm{~cm}^{-1}\right)$.

However, as was discussed above, the vertical design has weak mode confinement. In practice, up to 10-20 QDs layers with the same properties can be fabricated. It means that with vertical spacing $s=10 \mathrm{~nm}$ the total height of the structure cannot exceed $H_{\mathrm{A}} \sim 200 \mathrm{~nm}$, that is not enough for mode localization. So apart from this $H_{\mathrm{A}} \sim 200 \mathrm{~nm}$ of gain region the sandwiched structure should be complemented to $H \sim 1 \mu \mathrm{m}$ by another highindex material. Similar case was realized in [20], where MSM waveguide has $H_{\mathrm{A}}=300 \mathrm{~nm}$ of active gain region, while the whole ridge height $H>1 \mu \mathrm{m}$. It increases $\operatorname{Im}\left[k_{\text {eff }}\right]$ of the whole waveguide, therefore, any partial filling by active material increases propagation losses and suppresses efficiency of modulator.

Another solution is to increase vertical spacing $s$ and obtain relatively uniform distribution of gain medium along waveguide's cross section. However, in practice, it is hard to obtain vertical alignment at large spacing. Thus if we assume, in order to achieve $H_{\mathrm{A}} \sim 1 \mu \mathrm{m}$, spacing $s=50 \mathrm{~nm}$, the average gain falls down to $b_{\mathrm{d} 2}=0.9 \times 10^{3} \mathrm{~cm}^{-1}$.

\section{MSM waveguide with quantum wells}

As an alternative design to the MSM waveguide with a bulk semiconductor core we consider the MSM core which consists of several QWs separated by barriers (Fig. 9a). Tensile strained quantum wells are used to ensure a strong interaction with the electrical field of the mode. We took $\operatorname{In}_{0.466} \mathrm{Ga}_{0.534} \mathrm{As}$ QWs and $\mathrm{In}_{0.51} \mathrm{Ga}_{0.49} \mathrm{As}_{0.86} \mathrm{P}_{0.14}$ barriers with thicknesses $5 \mathrm{~nm}$. The composition is chosen such that to obtain the
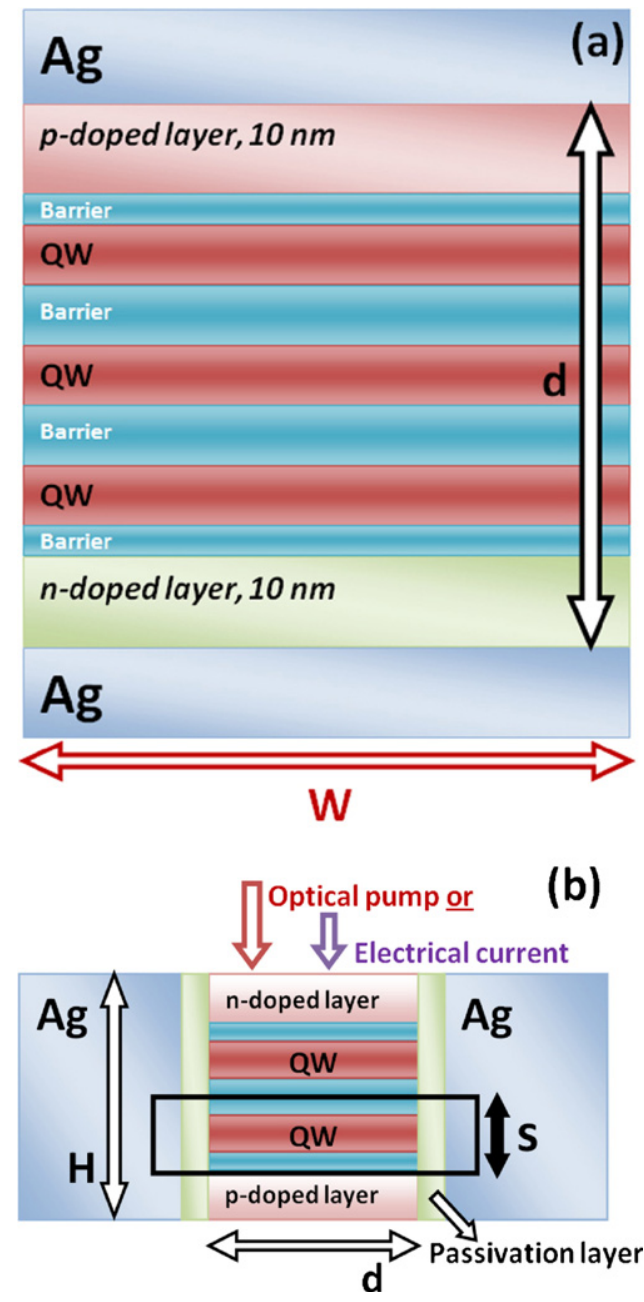

Fig. 9. Cross sections of the MSM waveguide with QWs. The MSM waveguide is composed of $\operatorname{In}_{0.466} \mathrm{Ga}_{0.534}$ As quantum wells stack, separated by $\operatorname{In}_{0.51} \mathrm{Ga}_{0.49} \mathrm{As}_{0.86} \mathrm{P}_{0.14}$ barriers with $\mathrm{n}$-doped InP layer and p-doped $\operatorname{In}_{0.53} \mathrm{Ga}_{0.47}$ As layer. (a) Horizontal design. (b) Vertical design.

bandgap at $1.55 \mu \mathrm{m}$ [47]. The wells are strain compensated, which allows stacking of many wells. However, due to the position of the barrier material in the miscibility gap, we decided to limit the number of wells to 25 . We performed simulations taking this number into account and defining the total core thickness based on it. In a QWs MSM system it is necessary to have at least one different medium with a higher energy band gap for the $\mathrm{n}$ - or $\mathrm{p}$-doped layer. Therefore, we choose 10 -nm thick $\operatorname{In}_{0.67} \mathrm{Ga}_{0.23}$ $\mathrm{As}_{0.71} \mathrm{P}_{0.29}$ layer and 10 -nm thick InP layer. As it was discussed in [22], adding a thin layer with low refractive index in between the waveguide and the metallic plates decreases the net gain in the system. This 


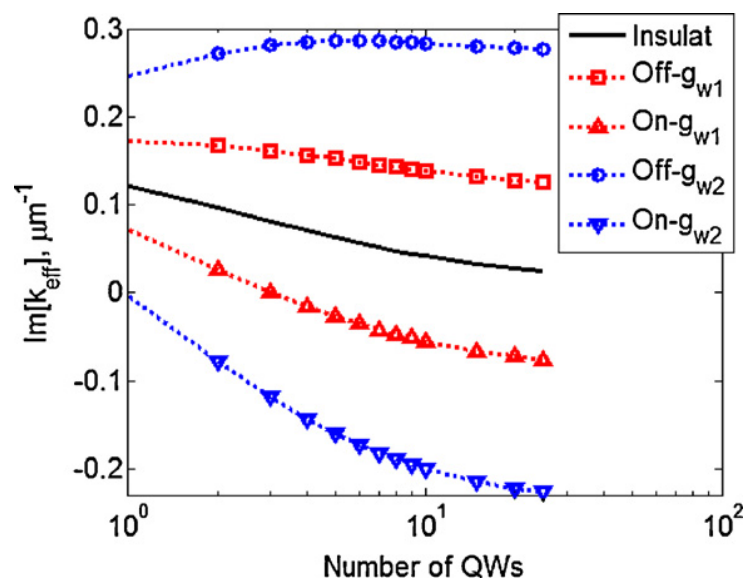

Fig. 10. Imaginary part of the effective propagation constant in the MSM waveguide with the QWs core. Notation: "Insulat" is passive waveguide, $g_{\mathrm{w} 1}=0.4 \times 10^{4} \mathrm{~cm}^{-1}$ and $g_{\mathrm{w} 2}=1 \times 10^{4} \mathrm{~cm}^{-1}$.

is due to confining the field outside the gain material, despite lowering the fields in the metallic layer.

The results of numerical simulations for $\operatorname{Im}\left[k_{\text {eff }}\right]$ and ER are shown in Figs. 10 and 11, respectively. We studied two gain levels, $g_{\mathrm{w} 1}=0.4 \times 10^{4} \mathrm{~cm}^{-1}$ and $g_{\mathrm{w} 2}=1 \times 10^{4} \mathrm{~cm}^{-1}$, which correspond to $\varepsilon^{\prime \prime}=-0.348$ and $\varepsilon^{\prime \prime}=-0.87$ respectively. The former $g_{\mathrm{w} 1}$ is currently achievable, e.g. in tensile QWs [37,48,49], while the latter $g_{\mathrm{w} 2}$ is feasible [50]. In such MSM waveguides with QWs complete loss compensation appears either for more than three QWs with gain $g_{\mathrm{w} 1}$ or already with one QW with gain $g_{\mathrm{w} 2}$. In contrast to QDs system, for the considered $g_{\mathrm{w} 1}$ and $g_{\mathrm{w} 2}$ values, ER of the QWs system is growing monotonously with the number of

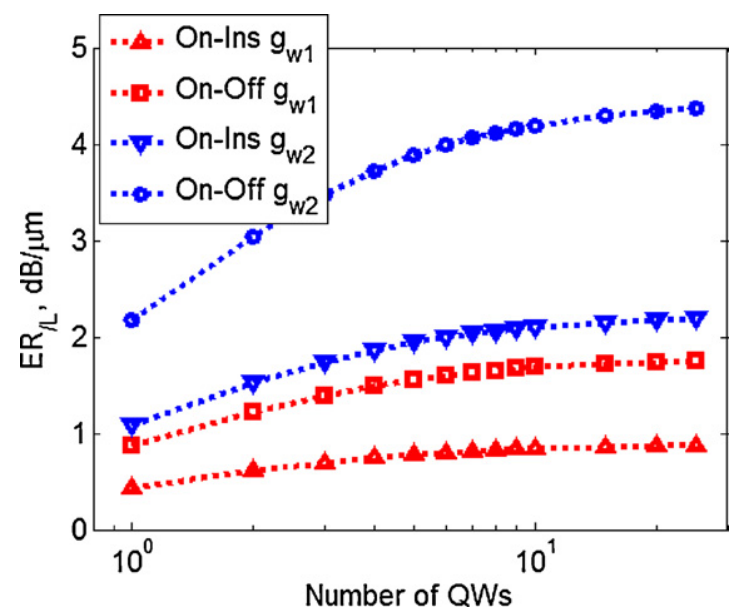

Fig. 11. ER of the MSM device with QWs. "On-Ins $g_{\mathrm{d} i}$ " shows transition between insulator-state $(g=0)$ and on-state with $g_{\mathrm{d} i}$. "OnOff $g_{\mathrm{d} i}$ " shows transition between off-state with $-g_{\mathrm{d} i}$ and on-state with $g_{\mathrm{d} i}\left(g_{\mathrm{w} 1}=0.4 \times 10^{4} \mathrm{~cm}^{-1}\right.$ and $\left.g_{\mathrm{w} 2}=1 \times 10^{4} \mathrm{~cm}^{-1}\right)$.
QWs and, starting at certain thicknesses (approximately $d=90 \mathrm{~nm}$ ), can be higher than in the case of the bulk semiconductor with $g_{\mathrm{b} 1}=0.1 \times 10^{4} \mathrm{~cm}^{-1}$.

Vertical design can be considered as well (Fig. 9b). The estimation of structure efficiency can be conducted as following. One period of the structure has thickness $s=10 \mathrm{~nm}$ and averaged gain $b_{\mathrm{w} 1}=0.2 \times 10^{4} \mathrm{~cm}^{-1}$ or $b_{\mathrm{w} 2}=0.5 \times 10^{4} \mathrm{~cm}^{-1}$, such that results for high gain of bulk medium can be applied. However, the total thickness of the gain region becomes $H_{\mathrm{A}} \sim 250 \mathrm{~nm}$, as the number of QWs is limited. To ensure mode localization, more than $400 \mathrm{~nm}$ thick layers of n- and pdoped semiconductor should be added as well, which increase $\operatorname{Im}\left[k_{\text {eff }}\right]$ and decrease device efficiency. Another option is the same as in the QDs system: the uniform distribution of QWs along the waveguide cross section can be applied by increasing the spacing between QWs.

\section{Discussion and conclusions}

We have considered three types of plasmonic modulators based on metal-semiconductor-metal waveguides with incorporating gain material. The switching principle is based on suppression of losses in plasmonic waveguides by gain material. The semiconductor core is studied in one of the three options: bulk semiconductor, QWs and layers with QDs. In our numerical simulations we considered realistic materials and applied gain parameters achieved in InGaAsP-based structures. We took different gain parameters considered in [37], including some that required very high carrier density. One of the reasons to do this is that we further apply bulk gain medium results for effective approximation of QDs and QWs structures.

We observe promising behavior in all cases: in principle it is possible not only to compensate losses, but also get waveguide mode amplification. The performance of the proposed modulators is greatly improved in comparison with plasmonic waveguides with controllable losses through electrically driven indium tin oxide layers. In practice, a passive MIM waveguide with $d=120 \mathrm{~nm}$ core thickness has $\operatorname{Im}\left[k_{\text {eff }}\right]=0.043 \mu \mathrm{m}^{-1}$. This means $15 \mathrm{~dB}$ attenuation on $40 \mu \mathrm{m}$ length. Following the fabrication requirements we have considered different layouts of the modulators with horizontal or vertical arrangement of gain layers. We can compare horizontal design with bulk semiconductor core and with QWs layers. A MSM waveguide with gain value $g_{\mathrm{b} 1}=0.1 \times 10^{4} \mathrm{~cm}^{-1}$ of bulk material and $10 \mathrm{~nm} \mathrm{n-} \mathrm{and} \mathrm{p-doped} \mathrm{passive} \mathrm{layers}$ gives slight amplification, i.e. $\operatorname{Im}\left[\mathrm{k}_{\mathrm{eff}}\right]=-0.007 \mu \mathrm{m}^{-1}$. 
Therefore, the dynamic range of the signal between passive and gain states in the $40 \mu \mathrm{m}$-long structure is estimated as $17.5 \mathrm{~dB}$, while between absorbing $\left(\operatorname{Im}\left[k_{\mathrm{eff}}\right]=0.093 \mu \mathrm{m}^{-1}\right)$ and gain states is doubled to $35 \mathrm{~dB}$. Assuming the same length for a QWs-based MSM waveguide in the horizontal arrangement $\left(g_{\mathrm{w} 1}=0.4 \times 10^{4} \mathrm{~cm}^{-1}\right.$ and $\left.\operatorname{Im}\left[k_{\mathrm{eff}}\right]=-0.055 \mu \mathrm{m}^{-1}\right)$ we obtained $34 \mathrm{~dB}$ depth of signal modulation between passive and gain states and $52 \mathrm{~dB}$ between absorbing and gain states. These results show that the QWs-based waveguides in the horizontal arrangement are preferable to the bulk based ones. Aside from that, QWs and QDs cores can be compared in vertical arrangement. QWs with $g_{\mathrm{w} 1}=0.4 \times 10^{4} \mathrm{~cm}^{-1}$ give effective bulk gain $b_{\mathrm{w} 1}=0.2 \times 10^{4} \mathrm{~cm}^{-1}$ of $10 \mathrm{~nm}$ thick active layer and QDs with $g_{\mathrm{d} 1}=1 \times 10^{4} \mathrm{~cm}^{-1}$ give effectively $b_{\mathrm{d} 1}=0.09 \times 10^{4} \mathrm{~cm}^{-1}$ because of small volume. Thus, both structures have approximately the same efficiency.

The FoM (4) of the InGaAsP-based plasmonic modulator is several times higher than the one for MIM plasmonic modulators with the silicon nitride and indium tin oxide multilayered core $[15,35,36]$. However, utilizing the studied MSM devices for modulation purposes encounters certain obstacles. The deep modulation requires a full switch of the current. Without enhancement of carrier recombination the response time is in the range of nanoseconds [37]. Nevertheless, modulation with speed up to $10 \mathrm{GHz}$ might be feasible, because of the inherent advantages of the MSM design, i.e. the small dimensions, strong coupling to the plasmonic structure, and enhancement of spontaneous emission due to the tight confinement of modes between two metal plates [51,52], that serves as electrodes.

Summarizing, all three designs of the MSM core exhibit effective switching. The preference in choosing the optimal design diverts from the pure numerical analysis of the device performance to the question of feasibility of fabrication such metal-semiconductor sandwiches. In addition, decision on vertical or horizontal arrangement of gain layers depends also on the waveguiding parameters, e.g. polarization and coupling efficiency.

Ultra-compact and ultra-fast modulators are among the main requirements for modern photonic integrated circuits. A surface plasmon polariton modulator supplemented with loss-compensation material provides such possibilities. Their potential applications range from direct laser modulation to on-chip optical routing and computation. For this, various in- and outcoupling schemes are needed. While for out-coupling to free space the solution can be straightforward the in-coupling from a VCSEL device is not necessary an easy task. A general in- and out-coupling scheme is difficult to suggest but we believe that, together with the development of plasmonic circuitry in photonics, the coupling problem will be solved.

\section{Acknowledgment}

R.M. and A.V.L. acknowledge partial financial support from the Danish Research Council for Technology and Production Sciences via the $\mathrm{THz}$ COW project.

\section{References}

[1] S.A. Maier, Plasmonics: Fundamentals and Applications, Springer Verlag, 2007.

[2] E. Ozbay, Science 311 (2006) 189-193.

[3] P. West, S. Ishii, G. Naik, N. Emani, V.M. Shalaev, A. Boltasseva, Laser and Photonics Reviews 4 (2010) 795-808.

[4] G.V. Naik, J. Kim, A. Boltasseva, Optical Materials Express 1 (2011) 1090-1099.

[5] M.A. Noginov, J. Lei Gu, G. Livenere, A.K. Zhu, R. Pradhan, M Mundle, Yu. Bahoura, A. Barnakov, V.A. Podolskiy, Applied Physics Letters 99 (2011) 021101.

[6] D.A. Bobb, G. Zhu, M. Mayy, A.V. Gavrilenko, P. Mead, V.I. Gavrilenko, M.A. Noginov, Applied Physics Letters 95 (2009) 151102 .

[7] M.P. Nezhad, K. Tetz, Y. Fainman, Optics Express 12 (2004) 4072-4079.

[8] I. Avrutsky, Physical Review B 70 (2004) 155416.

[9] S.A. Maier, Optics Communication 258 (2006) 295-299.

[10] S.M. García-Blanco, M. Pollnau, S.I. Bozhevolnyi, Optics Express 19 (2011) 25298-25311.

[11] D. Dai, Y. Shi, S. He, L. Wosinski, L. Thylen, Optics Express 19 (2011) 12925-12936.

[12] R. Zia, M.D. Selker, P.B. Catrysse, M.L. Brongersma, Journal of the Optical Society of America A: Optics and Image Science 21 (2004) 2442-2446.

[13] J.A. Dionne, H.J. Lezec, H.A. Atwater, Nano Letters 6 (2006) 1928-1932.

[14] J.A. Dionne, K. Diest, L.A. Sweatlock, H.A. Atwater, Nano Letters 9 (2009) 897-902.

[15] A. Melikyan, N. Lindenmann, S. Walheim, P.M. Leufke, S. Ulrich, J. Ye, P. Vincze, H. Hahn, Th. Schimmel, C. Koos, W. Freude, J. Leuthold, Optics Express 19 (2011) 8855-8869.

[16] P. Neutens, P. Van Dorpe, I. De Vlaminck, L. Lagae, G. Borghs, Nature Photonics 3 (2009) 283-286.

[17] P. Berini, I. De Leon, Nature Photonics 6 (2012) 16-24.

[18] D.B. Li, C.Z. Ning, Physical Review B 80 (2009) 153304.

[19] M.T. Hill, Y.-S. Oei, B. Smalbrugge, Y. Zhu, T. de Vries, P.J. van Veldhoven, F.W.M. van Otten, T.J. Eijkemans, J.S.P. Turkiewicz, H. de Waardt, E.J. Geluk, S.-H. Kwon, Y.-H. Lee, R. Nötzel, M.K. Smit, Nature Photonics 1 (2007) 589-594.

[20] M.T. Hill, M. Marell, E.S.P. Leong, B. Smalbrugge, Y. Zhu, M. Sun, P.J. van Veldhoven, E.J. Geluk, F. Karouta, Y.-S. Oei, R Nötzel, C.-Z. Ning, M.K. Smit, Optics Express 17 (2009) 11107-11112. 
[21] M.T. Hill, Journal of the Optical Society of America B: Optical Physics 27 (2010) B36-B44.

[22] X. Chen, B. Bhola, Y. Huang, S.T. Ho, Optics Express 18 (2010) $17220-17238$

[23] K.F. MacDonald, Z.L. Samson, M.I. Stockman, N.I. Zheludev, Nature Photonics 3 (2008) 55.

[24] K.F. MacDonald, N.I. Zheludev, Laser and Photonics Reviews 4 (2010) 562-567.

[25] D. Li, C.Z. Ning, Optics Express 19 (2011) 14594-14603.

[26] E.V. Ponizovskaya, A.M. Bratkovsky, Applied Physics A 95 (2009) 1137-1142.

[27] A.M. Bratkovsky, Proceedings of the IEEE 97 (2009) 13171328.

[28] C. Min, G. Veronis, Optics Express 17 (2009) 10757-10766.

[29] Y. Shen, G. Yu, J. Fu, L. Zou, Chinese Optics Letters 10 (2012) 021301.

[30] Z. Yu, G. Veronis, S. Fan, M.L. Brongersma, Applied Physics Letters 92 (2008) 041117.

[31] X. Gao, L. Ning, Optik - International Journal for Light and Electron Optics (2011), http://dx.doi.org/10.1016/j.ijleo. 2011.03.037.

[32] X. Mei, X.G. Huang, T. Jin, Plasmonics 6 (2011) 613-618.

[33] CST Microwave Studio, http://www.cst.com/.

[34] J. Geng, R.W. Ziolkowski, S. Campbell, R. Jin, X. Liang, Proc. IEEE AP-S Int. Symp., Spokane, WA, Paper 308.10, July, (2011), pp. 1624-1627.

[35] V.E. Babicheva, A.V. Lavrinenko, AIP Conference Proceedings 1398 (2011) 61-63.

[36] V.E. Babicheva, A.V. Lavrinenko, Proceedings of the SPIE 8424 (2012) 842413.

[37] L.A. Coldren, S.W. Corzine, Diode Lasers and Photonic Integrated Circuits, John Wiley \& Sons, New York, 1995.

[38] Yu.A. Goldberg, N.M. Schmidt, in: M. Levinshtein, S. Rumyantsev, M. Shur (Eds.), Handbook Series on
Semiconductor Parameters, vol. 2, World Scientific, London, 1999, pp. 153-179.

[39] V.M. Ustinov, N.A. Maleev, A.E. Zhukov, A.R. Kovsh, A.Yu. Egorov, et al. Applied Physics Letters 74 (1999) 2815.

[40] E.U. Rafailov, M.A. Cataluna, W. Sibbett, Nature Photonics 1 (2007) 395.

[41] E.S. Semenova, I.V. Kulkova, S. Kadkhodazadeh, M. Schubert, K. Yvind, Applied Physics Letters 99 (2011) 101106.

[42] S. Anantathanasarn, R. Noetzel, P.J. van Veldhoven, F.W.M. van Otten, T.J. Eijkemans, Y. Barbarin, T. de Vries, E. Smalbrugge, E.J. Geluk, E.A.J.M. Bente, Y.S. Oei, M.K. Smit, J.H. Wolter, Journal of Crystal Growth 298 (2007) 553.

[43] M. Asada, Y. Miyamoto, Y. Suernatsu, IEEE Journal of Quantum Electronics QE-22 (1986) 1915-1921.

[44] N. Kirstaedter, O.G. Schmidt, N.N. Ledentsov, D. Bimberg, V.M. Ustinov, A.Yu. Egorov, A.E. Zhukov, M.V. Maximov, P.S. Kop'ev, Zh.I. Alferov, Applied Physics Letters 69 (1996) 1226.

[45] K. Kawaguchi, N. Yasuoka, M. Ekawa, H. Ebe, T. Akiyama, M. Sugawara, Y. Arakawa, Applied Physics Letters 93 (2008) 121908.

[46] O.G. Schmidt, O. Kienzle, Y. Hao, K. Eberl, F. Ernst, Applied Physics Letters 74 (1999) 1272.

[47] I. Vurgaftman, J.R. Meyer, L.R. Ram-Mohan, Journal of Applied Physics 89 (2001) 5815.

[48] A.R. Adams, IEEE Journal of Selected Topics in Quantum Electronics 17 (2011) 1364-1373.

[49] J.D. Thomson, H.D. Summers, P.J. Hulyer, P.M. Smowton, P. Blood, Applied Physics Letters 75 (1999) 2527.

[50] P. Blood, IEEE Journal of Quantum Electronics 36 (2000) 354.

[51] Y.C. Jun, R.D. Kekatpure, J.S. White, M.L. Brongersma, Physical Review B 78 (2008) 153111-153121.

[52] J.A. Dionne, L.A. Sweatlock, M.T. Sheldon, A.P. Alivisatos, H.A. Atwater, IEEE Journal of Selected Topics in Quantum Electronics 16 (2010) 295-306. 